Research Article

\title{
Several Dynamical Properties for a Nonlinear Shallow Water Equation
}

\author{
Ls Yong and Haibo Yan \\ Department of Mathematics, Southwestern University of Finance and Economics, Chengdu 611130, China \\ Correspondence should be addressed to Haibo Yan; wengxiang00@163.com
}

Received 18 April 2014; Accepted 8 May 2014; Published 22 May 2014

Academic Editor: Gongnan Xie

Copyright (C) 2014 L. Yong and H. Yan. This is an open access article distributed under the Creative Commons Attribution License, which permits unrestricted use, distribution, and reproduction in any medium, provided the original work is properly cited.

A nonlinear third order dispersive shallow water equation including the Degasperis-Procesi model is investigated. The existence of weak solutions for the equation is proved in the space $L^{1}(R) \cap B V(R)$ under certain assumptions. The Oleinik type estimate and $L^{2 N}(R)$ ( $N$ is a natural number) estimate for the solution are obtained.

\section{Introduction}

Constantin and Lannes [1] derived the shallow water wave equation

$$
\begin{aligned}
u_{t} & +u_{x}+\frac{3}{2} \rho u u_{x}+\mu\left(\alpha u_{x x x}+\beta u_{x x t}\right) \\
& =\rho \mu\left(\gamma u_{x} u_{x x}+\delta u u_{x x x}\right),
\end{aligned}
$$

where the constants $\alpha, \beta, \gamma, \delta, \rho$, and $\mu$ satisfy certain conditions. As stated in [1], using suitable mathematical transformations, one can turn (1) into the form

$$
u_{t}-u_{x x t}+2 k u_{x}+m u u_{x}=a u_{x} u_{x x}+b u u_{x x x}
$$

where $a, b, k$, and $m$ are constants. Clearly, (2) contains both the Camassa-Holm and Degasperis-Procesi models.

The aim of this paper is to investigate the existence of weak solutions for the special case of (2). Namely, we study the shallow water equation

$$
\begin{array}{r}
\partial_{t} u-\partial_{t x x}^{3} u+m u \partial_{x} u=3 \partial_{x} u \partial_{x x}^{2} u+u \partial_{x x x}^{3} u \\
(t, x) \in R_{+} \times R,
\end{array}
$$

where $m>0$ is a constant. Letting $y=u-\partial_{x x}^{2} u, v=$ $\left(m-\partial_{x x}^{2}\right)^{-1} u$ and using (3), we derive the conservation law

$$
\begin{aligned}
\int_{R} y v d x & =\int_{R} \frac{1+\xi^{2}}{m+\xi^{2}}|\widehat{u}(\xi)|^{2} d \xi \\
& =\int_{R} \frac{1+\xi^{2}}{m+\xi^{2}}\left|\widehat{u}_{0}(\xi)\right|^{2} d \xi \sim\left\|u_{0}\right\|_{L^{2}(R)}
\end{aligned}
$$

where $u_{0}=u(0, x)$. In fact, the conservation law (4) takes an important role in our further investigations of (3).

For $m=4,(3)$ reduces to the Degasperis-Procesi equation $[2]$

$$
\begin{array}{r}
\partial_{t} u-\partial_{t x x}^{3} u+4 u \partial_{x} u=3 \partial_{x} u \partial_{x x}^{2} u+u \partial_{x x x}^{3} u \\
(t, x) \in R_{+} \times R,
\end{array}
$$

which has been studied by many scholars (see [3-5]). Lundmark and Szmigielski [6] developed an inverse scattering approach for computing $n$-peakon solutions to (5). The traveling wave solutions of (5) were investigated in Vakhnenko and Parkes [5]. Holm and Staley [7] studied stability of solitons and peakons numerically. Lin and Liu [8] proved the stability of peakons for the Degasperis-Procesi equation (5) under certain assumptions. The precise blowup scenario result, a blowup result, and the global existence of strong solutions and global weak solutions to (5) can be found in [9]. Matsuno [10] studied multisoliton solutions and their peakon limits. 
Analogous to the case of the Camassa-Holm equation, Henry [11] and Mustafa [12] showed that smooth solutions to (5) have infinite speed of propagation. For other methods to handle the problems relating to various dynamic properties of the Degasperis-Procesi equation and other shallow water equations, the reader is referred to [13-15] and the references therein.

Coclite and Karlsen [16] established the existence, uniqueness, and $L^{1}(R)$ stability of entropy weak solutions belonging to the class $L^{1}(R) \cap B V(R)$ for (5). They obtained existence of at least one weak solution satisfying a restricted set of entropy inequalities in the space $L^{2}(R) \cap L^{4}(R)$ and extended these results to a class of generalized DegasperisProcesi equations in [16].

Motivated by the desire to extend parts of the results presented in Coclite and Karlsen [16], we consider (3) with its Cauchy problem in the form

$$
\begin{aligned}
& \partial_{t} u-\partial_{t x x}^{3} u=-\partial_{x}\left(\frac{m}{2} u^{2}\right)+3 \partial_{x} u \partial_{x x}^{2} u+u \partial_{x x x}^{3} u \\
&=-\left(\frac{m}{2} u^{2}\right)_{x}+\frac{1}{2} \partial_{x x x}^{3} u^{2} \\
& u(0, x)=u_{0}(x)
\end{aligned}
$$

which is equivalent to

$$
\begin{gathered}
u_{t}+u u_{x}=-\frac{m-1}{2} \Lambda^{-2}\left(u^{2}\right)_{x} \\
u(0, x)=u_{0}(x)
\end{gathered}
$$

where $m>0$ is a constant and $\Lambda=\left(1-\partial_{x}^{2}\right)^{1 / 2}$.

The objective of this paper is to study (3). We investigate the existence of weak solutions in the space $L^{1}(R) \cap B V(R)$ under certain conditions. Several dynamical properties such as Oleinik type estimate and $L^{2 N}(R)$ ( $N$ is a natural number) are obtained. As (3) includes the Degasperis-Procesi equation (5), parts of results presented in [16] are extended. Here we should mention that the generalized Degasperis-Procesi equation discussed in [16] does not include the model (3). We state that the ideas and approaches to prove our main results come from those in [16].

The rest of this paper is organized as follows. Section 2 establishes the $L^{2}, B V$, and $L^{\infty}$ estimates for the viscous approximations of problem (6). The main result is given in Section 3

\section{Viscous Approximations and Estimates}

Firstly, we give some notations.

Set $R_{+}=[0,+\infty)$. The space of all infinitely differentiable functions $\phi(t, x)$ with compact support in $R_{+} \times R$ is denoted by $C_{0}^{\infty}$. We let $L^{p}=L^{p}(R)(1 \leq p<+\infty)$ be the space of all measurable functions $h$ such that $\|h\|_{L^{p}}^{p}=\int_{R}|h(t, x)|^{p} d x<$ $\infty$. We define $L^{\infty}=L^{\infty}(R)$ with the standard norm $\|h\|_{L^{\infty}}=\inf _{m(e)=0} \sup _{x \in R \backslash e}|h(t, x)|$. For any real number $s$, we let $H^{s}=H^{s}(R)$ denote the Sobolev space with the norm defined by

$$
\|h\|_{H^{s}}=\left(\int_{R}\left(1+|\xi|^{2}\right)^{s}|\widehat{h}(t, \xi)|^{2} d \xi\right)^{1 / 2}<\infty
$$

where $\widehat{h}(t, \xi)=\int_{R} e^{-i x \xi} h(t, x) d x$.

For $T>0$ and nonnegative number $s$, let $C\left([0, T) ; H^{s}(R)\right)$ denote the Frechet space of all continuous $H^{s}$-valued functions on $[0, T)$.

Defining

$$
\phi(x)= \begin{cases}e^{1 /\left(x^{2}-1\right)}, & |x|<1, \\ 0, & |x| \geq 1\end{cases}
$$

and letting $\phi_{\varepsilon}(x)=\varepsilon^{-1 / 4} \phi\left(\varepsilon^{-1 / 4} x\right)$ with $0<\varepsilon<1 / 4$ and $u_{0, \varepsilon}=\phi_{\varepsilon} \star u_{0}$, we know that $u_{0, \varepsilon} \in C^{\infty}$ for any $u_{0} \in H^{s}$ with $s \geq 0$.

For simplicity, throughout this paper, we let $c_{0}$ denote any positive constant, which is independent of parameter $\varepsilon$ and time $t$.

To establish the existence of solutions to the Cauchy problem (6), we will analyze the limiting behavior of a sequence of smooth functions $\left\{u_{\varepsilon}\right\}_{\varepsilon}>0$, where each function $u_{\varepsilon}$ satisfies the viscous problem

$$
\begin{gathered}
\partial_{t} u_{\varepsilon}-\partial_{t x x}^{3} u_{\varepsilon}+m u_{\varepsilon} \partial_{x} u_{\varepsilon} \\
=3 \partial_{x} u_{\varepsilon} \partial_{x x}^{2} u_{\varepsilon}+u_{\varepsilon} \partial_{x x x}^{3} u_{\varepsilon}+\varepsilon \partial_{x x}^{2} u_{\varepsilon}-\varepsilon \partial_{x x x x}^{4} u_{\varepsilon}, \\
(t, x) \in R_{+} \times R, \\
u_{\varepsilon}(0, x)=u_{0, \varepsilon}(x), \quad x \in R,
\end{gathered}
$$

which is equivalent to the parabolic-elliptic system

$$
\begin{gathered}
\partial_{t} u_{\varepsilon}+\partial_{x}\left(\frac{u_{\varepsilon}^{2}}{2}\right)+\partial_{x} P_{\varepsilon}=\varepsilon \partial_{x x} u_{\varepsilon}, \\
P_{\varepsilon}-\partial_{x x}^{2} P_{\varepsilon}=\frac{m-1}{2} u_{\varepsilon}^{2}, \\
u_{\varepsilon}(0, x)=u_{0, \varepsilon}(x) .
\end{gathered}
$$

From the second identity of (11), we get

$$
P_{\varepsilon}(t, x)=\frac{m-1}{4} \int_{R} e^{|x-y|} u_{\varepsilon}^{2}(t, y) d y .
$$

2.1. $L^{2}$ Estimates and Several Consequences. Several properties for the smooth function $u_{0, \varepsilon}$ are given in the following Lemma. 
Mathematical Problems in Engineering

3

Lemma 1. The following estimates hold for any $\varepsilon$ with $0<\varepsilon<$ $1 / 4$ and $s \geq 0$ :

$$
\begin{gathered}
\left\|u_{0, \varepsilon}\right\|_{L^{2}(R)} \leq c_{0}\left\|u_{0}\right\|_{L^{2}(R)}, \\
\left\|u_{0, \varepsilon}\right\|_{L^{1}(R)} \leq c_{0}\left\|u_{0}\right\|_{L^{1}(R)}, \\
\left\|u_{0, \varepsilon}\right\|_{B V(R)} \leq c_{0}\left\|u_{0}\right\|_{B V(R)}, \\
\left\|u_{0, \varepsilon}\right\|_{L^{p}(R)} \leq c_{0}\left\|u_{0}\right\|_{L^{p}(R)} \quad \text { for } 1 \leq p \leq \infty, \\
u_{0, \varepsilon} \longrightarrow u_{0} \quad(\varepsilon \longrightarrow 0) \text { in } L^{p}(R) \text { for } 1 \leq p \leq \infty, \\
\left\|u_{0, \varepsilon}\right\|_{H^{q}} \leq c_{0}\left\|u_{0}\right\|_{H^{s}}, \quad \text { if } q \leq s,
\end{gathered}
$$

where $c_{0}$ is a constant independent of $\varepsilon$.

The proof of Lemma 1 is similar to that of Lemma 5 presented in [14]. Here we omit it.

Lemma 2. Provided that $u_{0} \in L^{2}(R)$, for any fixed $\varepsilon>0$, there exists a unique global smooth solution $u_{\varepsilon}=u_{\varepsilon}(t, x)$ to the Cauchy problem (11) belonging to $C\left([0, \infty) ; H^{s}(R)\right)$ with $s \geq 0$.

Proof. We omit the proof since it is similar to the one found in [16] or [17] by using $u_{0, \varepsilon} \in C^{\infty}(R)$.

Lemma 3. Assume that $u_{0} \in L^{2}(R)$ holds and $u_{\varepsilon}$ is a solution of problem (10). Then, the following bounds hold for any $t \geq 0$ :

$$
\begin{gathered}
\left\|u_{\varepsilon}\right\|_{L^{2}} \leq c_{0}\left\|u_{0}\right\|_{L^{2}}, \\
\sqrt{\varepsilon}\left\|\partial_{x} u_{\varepsilon}\right\|_{L^{2}} \leq c_{0}\left\|u_{0}\right\|_{L^{2}},
\end{gathered}
$$

where $c_{0}$ is a positive constant independent of $\varepsilon$ and $t$.

Proof. Letting

$$
m v_{\varepsilon}-\partial_{x x}^{2} v_{\varepsilon}=u_{\varepsilon}
$$

derives

$$
v_{\varepsilon}=\left(m-\partial_{x x}^{2}\right)^{-1} u_{\varepsilon}
$$

Multiplying the first equation of problem (11) by $v_{\varepsilon}-\partial_{x x}^{2} v_{\varepsilon}$ and integrating over $R$ yield

$$
\begin{gathered}
\int_{R} \partial_{t} u_{\varepsilon}\left(v_{\varepsilon}-\partial_{x x}^{2} v_{\varepsilon}\right) d x-\varepsilon \int_{R} \partial_{x x}^{2} u_{\varepsilon}\left(v_{\varepsilon}-\partial_{x x}^{2} v_{\varepsilon}\right) d x \\
=-\int_{R} u_{\varepsilon} \partial_{x} u_{\varepsilon}\left(v_{\varepsilon}-\partial_{x x}^{2} v_{\varepsilon}\right) d x \\
-\int_{R} \partial_{x} P_{\varepsilon}\left(v_{\varepsilon}-\partial_{x x}^{2} v_{\varepsilon}\right) d x .
\end{gathered}
$$

For the left-hand side of this identity, using (16), we get

$$
\begin{aligned}
& \int_{R} \partial_{t} u_{\varepsilon}\left(v_{\varepsilon}-\partial_{x x}^{2} v_{\varepsilon}\right) d x-\varepsilon \int_{R} \partial_{x x}^{2} u_{\varepsilon}\left(v_{\varepsilon}-\partial_{x x}^{2} v_{\varepsilon}\right) d x \\
& =\int_{R}\left(m \partial_{t} v_{\varepsilon}-\partial_{t x x}^{3} v_{\varepsilon}\right)\left(v_{\varepsilon}-\partial_{x x}^{2} v_{\varepsilon}\right) d x \\
& -\varepsilon \int_{R}\left(m \partial_{x x}^{2} v_{\varepsilon}-\partial_{x x x x}^{4} v_{\varepsilon}\right)\left(v_{\varepsilon}-\partial_{x x}^{2} v_{\varepsilon}\right) d x \\
& =\int_{R}\left(m v_{\varepsilon} \partial_{t} v_{\varepsilon}-v_{\varepsilon} \partial_{t x x}^{3} v_{\varepsilon}-m \partial_{t} v_{\varepsilon} \partial_{x x}^{2} v_{\varepsilon}\right. \\
& \left.+\partial_{x x}^{2} v_{\varepsilon} \partial_{t x x}^{3} v_{\varepsilon}\right) d x \\
& -\varepsilon \int_{R}\left(m v_{\varepsilon} \partial_{x x}^{2} v_{\varepsilon}-m\left(\partial_{x x}^{2} v_{\varepsilon}\right)^{2}-v_{\varepsilon} \partial_{x x x x}^{4} v_{\varepsilon}\right. \\
& \left.+\partial_{x x}^{2} v_{\varepsilon} \partial_{x x x x}^{4} v_{\varepsilon}\right) d x \\
& =\int_{R}\left(m v_{\varepsilon} \partial_{t} v_{\varepsilon}-(m+1) v_{\varepsilon} \partial_{t x x}^{3} v_{\varepsilon}+\partial_{x x}^{2} v_{\varepsilon} \partial_{t x x}^{3} v_{\varepsilon}\right) d x \\
& -\varepsilon \int_{R}\left(m v_{\varepsilon} \partial_{x x}^{2} v_{\varepsilon}-(m+1) v_{\varepsilon} \partial_{x x x x}^{4} v_{\varepsilon}\right. \\
& \left.+\partial_{x x}^{2} v_{\varepsilon} \partial_{x x x x}^{4} v_{\varepsilon}\right) d x \\
& =\int_{R}\left(m v_{\varepsilon} \partial_{t} v_{\varepsilon}+(m+1) \partial_{x} v_{\varepsilon} \partial_{t x}^{2} v_{\varepsilon}+\partial_{x x}^{2} v_{\varepsilon} \partial_{t x x}^{3} v_{\varepsilon}\right) d x \\
& -\varepsilon \int_{R}\left(-m \partial_{x} v_{\varepsilon} \partial_{x} v_{\varepsilon}-(m+1) \partial_{x x}^{2} v_{\varepsilon} \partial_{x x}^{2} v_{\varepsilon}\right. \\
& \left.-\partial_{x x x}^{3} v_{\varepsilon} \partial_{x x x}^{3} v_{\varepsilon}\right) d x \\
& =\frac{1}{2} \frac{d}{d t} \int_{R}\left(m v_{\varepsilon}^{2}+(m+1)\left(\partial_{x} v_{\varepsilon}\right)^{2}+\left(\partial_{x x}^{2} v_{\varepsilon}\right)^{2}\right) d x \\
& +\varepsilon \int_{R}\left(m\left(\partial_{x} v_{\varepsilon}\right)^{2}+(m+1)\left(\partial_{x x}^{2} v_{\varepsilon}\right)^{2}+\left(\partial_{x x x}^{3} v_{\varepsilon}\right)^{2}\right) d x .
\end{aligned}
$$

For the right-hand side of (18), we conclude

$$
\begin{aligned}
- & \int_{R} u_{\varepsilon} \partial_{x} u_{\varepsilon}\left(v_{\varepsilon}-\partial_{x x}^{2} v_{\varepsilon}\right) d x-\int_{R} \partial_{x} P_{\varepsilon}\left(v_{\varepsilon}-\partial_{x x}^{2} v_{\varepsilon}\right) d x \\
= & -\int_{R} u_{\varepsilon} \partial_{x} u_{\varepsilon}\left(v_{\varepsilon}-\partial_{x x}^{2} v_{\varepsilon}\right) d x \\
& +\int_{R}\left(P_{\varepsilon}-\partial_{x x}^{2} P_{\varepsilon}\right) \partial_{x} v_{\varepsilon} d x \\
= & -\int_{R} u_{\varepsilon} \partial_{x} u_{\varepsilon}\left(v_{\varepsilon}-\partial_{x x}^{2} v_{\varepsilon}\right) d x
\end{aligned}
$$




$$
\begin{aligned}
& +\frac{m-1}{2} \int_{R} u_{\varepsilon}^{2} \partial_{x} v_{\varepsilon} d x \\
= & -\int_{R} u_{\varepsilon} \partial_{x} u_{\varepsilon}\left(v_{\varepsilon}-\partial_{x x}^{2} v_{\varepsilon}\right) d x \\
& -(m-1) \int_{R} u_{\varepsilon} \partial_{x} u_{\varepsilon} v_{\varepsilon} d x \\
= & \int_{R}\left(-m u_{\varepsilon} \partial_{x} u_{\varepsilon} v_{\varepsilon}+u_{\varepsilon} \partial_{x} u_{\varepsilon} \partial_{x x}^{2} v_{\varepsilon}\right) d x \\
= & \int_{R} u_{\varepsilon} \partial_{x} u_{\varepsilon}\left(-m v_{\varepsilon}+\partial_{x x}^{2} v_{\varepsilon}\right) d x \\
= & -\int_{R} u_{\varepsilon}^{2} \partial_{x} u_{\varepsilon} d x \\
= & 0,
\end{aligned}
$$

where we have used (16) and integration by parts.

From (18), (19), and (20), we have

$$
\begin{gathered}
m\left\|v_{\varepsilon}\right\|_{L^{2}}^{2}+(m+1)\left\|\partial_{x} v_{\varepsilon}\right\|_{L^{2}}^{2}+\left\|\partial_{x x}^{2} v_{\varepsilon}\right\|_{L^{2}}^{2} \\
+2 \varepsilon \int_{R}\left(m\left\|\partial_{x} v_{\varepsilon}\right\|_{L^{2}}^{2}+(m+1)\left\|\partial_{x x}^{2} v_{\varepsilon}\right\|_{L^{2}}^{2}\right. \\
\left.+\left\|\partial_{x x x}^{3} v_{\varepsilon}\right\|_{L^{2}}^{2}\right) d x \\
=m\left\|v_{\varepsilon}(0, \cdot)\right\|_{L^{2}}^{2}+(m+1)\left\|\partial_{x} v_{\varepsilon}(0, \cdot)\right\|_{L^{2}}^{2} \\
+\left\|\partial_{x x}^{2} v_{\varepsilon}(0, \cdot)\right\|_{L^{2}}^{2} .
\end{gathered}
$$

From (17), we obtain

$$
\begin{aligned}
& \left\|v_{\varepsilon}(0, \cdot)\right\|_{L^{2}},\left\|\partial_{x} v_{\varepsilon}(0, \cdot)\right\|_{L^{2}},\left\|\partial_{x x}^{2} v_{\varepsilon}(0, \cdot)\right\|_{L^{2}} \\
& \quad \leq c_{0}\left\|u_{0, \varepsilon}\right\|_{L^{2}} \leq c_{0}\left\|u_{0}\right\|_{L^{2}} .
\end{aligned}
$$

It follows from (16) that

$$
\begin{aligned}
\left\|u_{\varepsilon}(t, \cdot)\right\|_{L^{2}(R)}^{2} & \\
= & \int_{R}\left(-\partial_{x x}^{2} v_{\varepsilon}+m v_{\varepsilon}\right)^{2} d x \\
= & \int_{R}\left(\partial_{x x}^{2} v_{\varepsilon}\right)^{2} d x-2 m \int_{R} v_{\varepsilon} \partial_{x x}^{2} v_{\varepsilon} d x \\
& +m^{2} \int_{R} v_{\varepsilon}^{2} d x \\
= & \int_{R}\left(\partial_{x x}^{2} v_{\varepsilon}\right)^{2} d x+2 m \int_{R}\left(\partial_{x} v_{\varepsilon}\right)^{2} d x \\
& +m^{2} \int_{R} v_{\varepsilon}^{2} d x .
\end{aligned}
$$

Using (16), (21), and Lemma 1 derives that

$$
\begin{aligned}
& \left\|u_{\varepsilon}\right\|_{L^{2}}^{2} \\
& \quad \leq 2\left\|\partial_{x x}^{2} v_{\varepsilon}\right\|_{L^{2}}^{2}+2 m^{2}\left\|v_{\varepsilon}\right\|_{L^{2}}^{2} \\
& \quad \leq \max (2,2 m)\left(m\left\|v_{\varepsilon}\right\|_{L^{2}}^{2}+(m+1)\left\|\partial_{x} v_{\varepsilon}\right\|_{L^{2}}^{2}\right. \\
& \left.\quad+\left\|\partial_{x x}^{2} v_{\varepsilon}\right\|_{L^{2}}^{2}\right) \\
& \quad \leq \max (2,2 m)\left(m\left\|v_{\varepsilon}(0, \cdot)\right\|_{L^{2}}^{2}+(m+1)\left\|\partial_{x} v_{\varepsilon}(0, \cdot)\right\|_{L^{2}}^{2}\right. \\
& \left.\quad+\left\|\partial_{x x}^{2} v_{\varepsilon}(0, \cdot)\right\|_{L^{2}}^{2}\right) \\
& \left.\quad \leq c_{0}\left\|u_{0, \varepsilon}\right\|_{L^{2}}^{2}\left\|_{L^{2}}^{2}, \quad+\right\| \partial_{x x}^{2} v_{\varepsilon}(0, \cdot) \|_{L^{2}}^{2}\right) \\
& \left.\quad \leq c_{x_{0}} u_{\varepsilon}\left\|_{L^{2}}^{2}\right\| u_{0}\left\|_{L^{2}}^{2} \cdot \quad+\right\| \partial_{x x x}^{3} v_{\varepsilon} \|_{L^{2}}^{2}\right) \\
& \quad \leq 2 \varepsilon\left\|\partial_{x x x}^{3} v_{\varepsilon}\right\|_{L^{2}}^{2}+2 m\left\|_{L^{2} \varepsilon}^{2}\right\| \partial_{x} v_{\varepsilon} \|_{L^{2}}^{2} \\
& \quad \leq \varepsilon \max (2,2 m)\left(m\left\|\partial_{x} v_{\varepsilon}\right\|_{L^{2}}^{2}+(m+1)\left\|\partial_{x x}^{2} v_{\varepsilon}\right\|_{L^{2}}^{2}\right.
\end{aligned}
$$

The proof of Lemma 3 follows from (24).

We give some bounds on the nonlocal term $P_{\varepsilon}$, in which all are consequences of the $L^{2}$ bound in Lemma 3.

Lemma 4. Assume that $u_{0} \in L^{2}(R)$ holds. Then,

$$
P_{\varepsilon} \geq 0 \quad \text { for } m \geq 1 \text {, }
$$

$\left\|P_{\varepsilon}(t, \cdot)\right\|_{L^{1}(R)}, \quad\left\|\partial_{x} P_{\varepsilon}(t, \cdot)\right\|_{L^{1}(R)} \leq c_{0} \frac{|m-1|}{2}\left\|u_{0}\right\|_{L^{2}}^{2}$,

$$
\begin{gathered}
\left\|P_{\varepsilon}\right\|_{L^{\infty}\left(R_{+} \times R\right)}, \quad\left\|\partial_{x} P_{\varepsilon}\right\|_{L^{\infty}\left(R_{+} \times R\right)} \leq c_{0} \frac{|m-1|}{2}\left\|u_{0}\right\|_{L^{2}}^{2}, \\
\left\|\partial_{x x}^{2} P_{\varepsilon}(t, \cdot)\right\|_{L^{1}(R)} \leq c_{0} \frac{|m-1|}{2}\left\|u_{0}\right\|_{L^{2}}^{2}
\end{gathered}
$$

where $c_{0}$ is a constant independent of $\varepsilon$ and $t$. 
Proof. Using (11), we get

$$
\begin{gathered}
P_{\varepsilon}(t, x)=\frac{m-1}{4} \int_{R} e^{-|x-y|}\left(u_{\varepsilon}(t, y)\right)^{2} d y \\
\partial_{x} P_{\varepsilon}(t, x)=\frac{m-1}{4} \int_{R} e^{-|x-y|} \operatorname{sign}(y-x)\left(u_{\varepsilon}(t, y)\right)^{2} d y .
\end{gathered}
$$

From (29), we obtain (25). Using (14) and the Tonelli theorem, we have

$$
\begin{aligned}
& \int_{R}\left|P_{\varepsilon}(t, x)\right| d x \\
& \leq \frac{|m-1|}{4} \int_{R}\left(\int_{R} e^{-|x-y|} d x\right)\left(u_{\varepsilon}(t, y)\right)^{2} d y \\
& \leq c_{0} \frac{|m-1|}{2} \int_{R}\left(u_{\varepsilon}(t, y)\right)^{2} d y \\
& \leq c_{0} \frac{|m-1|}{2}\left\|u_{0}\right\|_{L^{2}}^{2}, \\
& \int_{R}\left|\partial_{x} P_{\varepsilon}(t, x)\right| d x \\
& \leq \frac{|m-1|}{4} \int_{R}\left(\int_{R} e^{-|x-y|} d x\right)\left(u_{\varepsilon}(t, y)\right)^{2} d y \\
& \leq c_{0} \frac{|m-1|}{2} \int_{R}\left(u_{\varepsilon}(t, y)\right)^{2} d y \\
& \leq c_{0} \frac{|m-1|}{2}\left\|u_{0}\right\|_{L^{2}}^{2}, \\
& \left|P_{\varepsilon}(t, x)\right| \leq \frac{|m-1|}{4} \int_{R}\left(u_{\varepsilon}(t, y)\right)^{2} d y \\
& \leq c_{0} \frac{|m-1|}{4}\left\|u_{0}\right\|_{L^{2}}^{2}, \\
& \left|\partial_{x} P_{\varepsilon}(t, x)\right| \leq \frac{|m-1|}{4} \int_{R}\left(u_{\varepsilon}(t, y)\right)^{2} d y \\
& \leq c_{0} \frac{|m-1|}{4}\left\|u_{0}\right\|_{L^{2}}^{2} \text {. }
\end{aligned}
$$

It follows from (31) that (26) and (27) hold. Using the second identity of problem (11), Lemma 3, and (26), we obtain (28).

Lemma 5. If $u_{0} \in L^{1}(R) \cap L^{2}(R)$, it holds that

$$
\left\|u_{\varepsilon}(t, \cdot)\right\|_{L^{1}} \leq\left\|u_{0}\right\|_{L^{1}}+c_{0} t\left\|u_{0}\right\|_{L^{2}}^{2}
$$

Proof. Let functions $\eta$ and $q: R \rightarrow R$ be such that $q^{\prime}(u)=$ $u \eta^{\prime}(u)$. Multiplying the first equation in (11) with $\eta^{\prime}\left(u_{\varepsilon}\right)$ gives rise to

$$
\begin{aligned}
\partial_{t} \eta & \left(u_{\varepsilon}\right)+\partial_{x} q\left(u_{\varepsilon}\right)+\eta^{\prime}\left(u_{\varepsilon}\right) \partial_{x} P_{\varepsilon} \\
= & \varepsilon \eta^{\prime}\left(u_{\varepsilon}\right) \partial_{x x}^{2} u_{\varepsilon} \\
& =\varepsilon \partial_{x x}^{2} \eta\left(u_{\varepsilon}\right)-\varepsilon \eta^{\prime \prime}\left(u_{\varepsilon}\right)\left(\partial_{x} u_{\varepsilon}\right)^{2} .
\end{aligned}
$$

Choosing $\eta(u)=|u|$ (modulo an approximation argument, see [16]) and then integrating the resulting equation over $R$ yield

$$
\frac{d}{d t} \int_{R}\left|u_{\varepsilon}\right| d x \leq \int_{R}\left|\operatorname{sign}\left(u_{\varepsilon}\right) \partial_{x} P_{\varepsilon}\right| d x
$$

Using (26), we get

$$
\frac{d}{d t}\left\|u_{\varepsilon}(t, \cdot)\right\|_{L^{1}} \leq c_{0} \frac{|m-1|}{2}\left\|u_{0}\right\|_{L^{2}}^{2},
$$

from which we have (32).

2.2. $B V$ and $L^{\infty}$ Estimates. In this subsection we establish several supplementary estimates for the viscous approximations, which also are consequences of the $L^{2}$ bound in Lemma 3. In particular, we prove that the sequence $\left\{u_{\varepsilon}\right\}_{\varepsilon>0}$ is bounded in $B V$, which yields strong compactness of this sequence. To this end, we need to assume that $u_{0} \in L^{2}(R)$ and $u_{0} \in B V(R)$.

Lemma 6. Assume that $u_{0} \in L^{2}(R)$ and $u_{0} \in B V(R)$ hold. Then,

$$
\left\|\partial_{x} u_{\varepsilon}(t, \cdot)\right\|_{L^{1}(R)} \leq c_{0}\left(\left|u_{0}\right|_{B V}+t\left\|u_{0}\right\|_{L^{2}}^{2}\right), \quad t \geq 0 .
$$

Proof. Setting $q_{\varepsilon}:=\partial_{x} u_{\varepsilon}$, we know that $q_{\varepsilon}$ satisfies the equation

$$
\partial_{t} q_{\varepsilon}+u_{\varepsilon} \partial_{x} q_{\varepsilon}+q_{\varepsilon}^{2}+\partial_{x x}^{2} P_{\varepsilon}=\varepsilon \partial_{x x}^{2} q_{\varepsilon} .
$$

If $\eta=\eta(u)$ and $q: R \rightarrow R$ satisfies $q^{\prime}(u)=u \eta^{\prime}(u)$, using the chain rule yields

$$
\begin{gathered}
\partial_{t} \eta\left(q_{\varepsilon}\right)+\partial_{x}\left(u_{\varepsilon} q\left(u_{\varepsilon}\right)\right)-q_{\varepsilon} \eta\left(q_{\varepsilon}\right) \\
+\eta^{\prime}\left(q_{\varepsilon}\right) q_{\varepsilon}^{2}+\eta^{\prime}\left(u_{\varepsilon}\right) \partial_{x x}^{2} P_{\varepsilon} \\
=\varepsilon \partial_{x x}^{2} \eta\left(q_{\varepsilon}\right)-\varepsilon \eta^{\prime \prime}\left(q_{\varepsilon}\right)\left(\partial_{x} q_{\varepsilon}\right)^{2} .
\end{gathered}
$$

Choosing $\eta(u)=|u|$ (modulo an approximation argument) and then integrating the resulting equation over $R$ give rise to

$$
\frac{d}{d t} \int_{R}\left|\partial_{x} u_{\varepsilon}\right| d x \leq \int_{R}\left|\partial_{x x}^{2} P_{\varepsilon}\right| d x
$$

Using (28), we have

$$
\frac{d}{d t} \int_{R}\left|\partial_{x} u_{\varepsilon}\right| d x \leq c_{0}\left\|u_{0}\right\|_{L^{2}(R)}^{2},
$$

from which we obtain (36).

Lemma 7. Assume that $u_{0} \in B V(R) \cap L^{2}(R)$ holds. Then,

$$
\begin{gathered}
\left\|u_{\varepsilon}(t, \cdot)\right\|_{L^{\infty}(R)} \leq c_{0}\left(\left|u_{0}\right|_{B V(R)}+t\left\|u_{0}\right\|_{L^{2}(R)}^{2}\right), \\
t \geq 0, \\
\left\|\partial_{x x}^{2} P_{\varepsilon}(t, \cdot)\right\|_{L^{\infty}} \\
\quad \leq c_{0}\left[\left\|u_{0}\right\|_{L^{2}(R)}^{2}+\left(\left|u_{0}\right|_{B V(R)}+t\left\|u_{0}\right\|_{L^{2}(R)}\right)^{2}\right],
\end{gathered}
$$

where $c_{0}$ is independent of $\varepsilon$ and $t$. 
Proof. Using

$$
\left|u_{\varepsilon}(t, \cdot)\right| \leq \int_{R}\left|\partial_{x} u_{\varepsilon}(t, y)\right| d y=\left\|u_{\varepsilon}(t, \cdot)\right\|_{B V(R)}
$$

and Lemma 6 derives (41). Using (27), (41), and the second equation of problem (11), we obtain inequality (42).

2.3. $L^{2 N}$ Estimate for Nature Number $N \geq 2$. Next we prove that the viscous approximations are bounded in $L^{2 N}(R)$ for any nature number $N \geq 2$. From Lemmas 6 and 7, if $u_{0} \in$ $B V(R) \cap L^{2}(R)$, we have the inequality $u_{0, \varepsilon} \in L^{\infty}(R)$ from which we derive

$$
u_{0, \varepsilon} \in L^{2}(R) \bigcap L^{2 N}(R)
$$

Lemma 8. Assume that $u_{0} \in B V(R) \cap L^{2}(R)$ holds. For any $0<\varepsilon<1 / 4$, it has

$$
\begin{aligned}
\left\|u_{\varepsilon}(t, \cdot)\right\|_{L^{2 N}(R)} \leq & c_{0}\left(1+\left\|u_{0}\right\|_{L^{2}}^{2}\right)^{2} t \\
& +\left\|u_{\varepsilon}(0, \cdot)\right\|_{L^{2 N}(R)}^{2 N} e^{c_{0}\left(1+\left\|u_{0}\right\|_{L^{2}}^{2}\right)^{2} t} .
\end{aligned}
$$

Proof. Choosing $\eta(u)=(1 / 2 N) u^{2 N}$ in (33), writing

$$
\begin{aligned}
\partial_{t} \eta & \left(u_{\varepsilon}\right)+\partial_{x} q\left(u_{\varepsilon}\right)+\eta^{\prime}\left(u_{\varepsilon}\right) \partial_{x} P_{\varepsilon} \\
& =\varepsilon \partial_{x x}^{2} \eta\left(u_{\varepsilon}\right)-\varepsilon \eta^{\prime \prime}\left(u_{\varepsilon}\right)\left(\partial_{x} u_{\varepsilon}\right)^{2} \\
& =\varepsilon \eta^{\prime}\left(u_{\varepsilon}\right) \partial_{x x}^{2} u_{\varepsilon} \\
& =\varepsilon u_{\varepsilon}^{2 N-1} \partial_{x x}^{2} u_{\varepsilon},
\end{aligned}
$$

and integrating (46) over $R$ gives rise to

$$
\begin{aligned}
\frac{1}{2 N} \frac{d}{d t}\left\|u_{\varepsilon}(t, \cdot)\right\|_{L^{2 N}}^{2 N}= & -\int_{R} u_{\varepsilon}^{2 N-1} \partial_{x} P_{\varepsilon} d x \\
& +\varepsilon \int_{R} u_{\varepsilon}^{2 N-1} \partial_{x x}^{2} u_{\varepsilon} d x
\end{aligned}
$$

Integration by parts shows

$$
\begin{aligned}
& \varepsilon \int_{R} u_{\varepsilon}^{2 N-1} \partial_{x x}^{2} u_{\varepsilon} d x \\
& \quad=-(2 N-1) \varepsilon \int_{R} u_{\varepsilon}^{(2 N-2)}\left(\partial_{x} u_{\varepsilon}\right)^{2} d x \leq 0 .
\end{aligned}
$$

Letting $p_{1}=2 N /(2 N-1), q_{1}=2 N$, we have $1 / p_{1}+1 / q_{1}=1$. Using Hölder's inequality, (26), and (27), we obtain

$$
\begin{aligned}
\mid \int_{R} & u_{\varepsilon}^{2 N-1} \partial_{x} P_{\varepsilon} d x \mid \\
\leq & \left(\int_{R} u_{\varepsilon}^{2 N} d x\right)^{(2 N-1) / 2 N}\left(\int_{R}\left(\partial_{x} P_{\varepsilon}\right)^{2 N}\right)^{1 / 2 N} \\
\leq & \left(1+\int_{R} u_{\varepsilon}^{2 N} d x\right)^{(2 N-1) / 2 N} \\
& \times\left(\left\|\partial_{x} P_{\varepsilon}\right\|_{L^{\infty}}^{2 N-1}\left(R_{+} \times R\right)\right)^{1 / 2 N}\left\|\partial_{x} P_{\varepsilon}\right\|_{L^{1}(R)}^{1 / 2 N} \\
\leq & \left(1+\int_{R} u_{\varepsilon}^{2 N} d x\right)\left(1+\left\|\partial_{x} P_{\varepsilon}\right\|_{L^{\infty}\left(R_{+} \times R\right)}\right)\left\|\partial_{x} P_{\varepsilon}\right\|_{L^{1}(R)}^{1 / 2 N} \\
\leq & c_{0}\left(1+\left\|u_{0}\right\|_{L^{2}}^{2}\right)\left(1+\left\|u_{0}\right\|_{L^{2}}^{2}\right)^{1 / 2 N}\left(1+\int_{R} u_{\varepsilon}^{2 N} d x\right) \\
\leq & c_{0}\left(1+\left\|u_{0}\right\|_{L^{2}}^{2}\right)^{2}\left(1+\int_{R} u_{\varepsilon}^{2 N} d x\right) .
\end{aligned}
$$

Using (47)-(49) gives rise to

$$
\begin{aligned}
& \frac{1}{2 N} \frac{d}{d t}\left\|u_{\varepsilon}(t, \cdot)\right\|_{L^{2 N}}^{2 N} \\
& \quad \leq c_{0}\left(1+\left\|u_{0}\right\|_{L^{2}}^{2}\right)^{2}\left(1+\left\|u_{\varepsilon}(t, \cdot)\right\|_{L^{2 N}}^{2 N}\right),
\end{aligned}
$$

from which we obtain (45) by Gronwall's inequality.

\subsection{Oleinik Type Estimate}

Lemma 9 (Oleinik type estimate). Assume that $u_{0} \in L^{2}(R) \cap$ $B V(R)$. Then, for each $t \in(0, T]$ with $T$ being fixed,

$$
\partial_{x} u_{\varepsilon}(t, x) \leq \frac{1}{t}+K_{T}, \quad x \in R,
$$

where $K_{T}=c_{0}\left\{\left\|u_{0}\right\|_{L^{2}(R)}^{2}+\left(\left\|u_{0}\right\|_{B V(R)}+T\left\|u_{0}\right\|_{L^{2}(R)}^{2}\right)^{2}\right\}^{1 / 2}$.

Proof. Setting $q_{\varepsilon}:=\partial_{x} u_{\varepsilon}$, it follows from (11) and (42) that

$$
\partial_{t} q_{\varepsilon}+u_{\varepsilon} \partial_{x} q_{\varepsilon}+q_{\varepsilon}^{2}-\varepsilon \partial_{x x}^{2} q_{\varepsilon}=-\partial_{x x}^{2} P_{\varepsilon} \leq K_{T}^{2} .
$$

Considering the ordinary differential equation

$$
\frac{d f}{d t}+f^{2}=K_{T}^{2}
$$

and using comparing theorem, we have

$$
\partial_{x} u_{\varepsilon}(t, x) \leq \frac{1}{t}+K_{T}, \quad x \in R,
$$

which completes the proof.

\section{Existence in $L^{1}(R) \cap B V(R)$}

Using the estimates established in Section 2, we will show the existence of weak solutions to problem (6) under the assumption $u_{0} \in L^{1}(R) \cap B V(R)$.

We state the concepts of weak solutions. 
Definition 10 (weak solution). We call a function $u: R_{+} \times$ $R \rightarrow R$ a weak solution of the Cauchy problem (6) provided that

(i) $u \in L^{\infty}\left(R_{+} ; L^{2}(R)\right)$ and

(ii) $\partial_{t} u+\partial_{x}\left(u^{2} / 2\right)+\partial_{x} P^{u}(t, x)=0$ in $D^{\prime}([0, \infty) \times R)$; that is, for all $\phi \in C_{0}^{\infty}([0, \infty) \times R)$, there holds the identity

$$
\begin{aligned}
& \int_{R^{+}} \int_{R}\left(u \partial_{t} \phi+\frac{u^{2}}{2} \partial_{x} \phi-\partial_{x} P^{u} \phi\right) d x d t \\
& +\int_{R} u_{0}(x) \phi(0, x) d x=0,
\end{aligned}
$$

where

$$
\begin{aligned}
P^{u}(t, x) & =G_{1} *\left(\frac{m-1}{2} u^{2}\right)(t, x) \\
& =\frac{m-1}{4} \int_{R} e^{-|x-y|}(u(t, y))^{2} d y .
\end{aligned}
$$

Remark 11. It follows from part (i) of Definition 10 that $u \in$ $L^{1}((0, T) \times R)$ for any $T>0$ and $\partial_{x} P^{u} \in L^{\infty}\left(R_{+} \times R\right)$ (see Lemma 5). Therefore, (55) makes sense.

We assume that

$$
\begin{array}{r}
\left\|u\left(t_{2}, \cdot\right)-u\left(t_{1}, \cdot\right)\right\|_{L^{1}(R)} \leq C_{T}\left|t_{2}-t_{1}\right| \\
\text { for } \forall t_{1}, t_{2} \in[0, T],
\end{array}
$$

where $C_{T}=c_{0}\left[\left\|u_{0}\right\|_{L^{2}(R)}^{2}+\left(\left|u_{0}\right|_{B V(R)}+t\left\|u_{0}\right\|_{L^{2}(R)}\right)^{2}\right]$. Therefore, we have

$$
\lim _{t \rightarrow 0+}\left\|u(t, \cdot)-u_{0}\right\|_{L^{1}(R)}=0 .
$$

Our main results are summarized in the following Theorem.

Theorem 12. Provided that $u_{0} \in L^{1}(R) \cap L^{2}(R) \cap B V(R)$ and the solution $u(t, x)$ satisfies (57), then there exists a weak solution to the Cauchy problem (6). The weak solution $u$ satisfies the following estimates for any $t \in(0, T)$ :

$$
\|u(t, \cdot)\|_{L^{1}(R)} \leq\left\|u_{0}\right\|_{L^{1}}+c_{0} t\left\|u_{0}\right\|_{L^{2}}^{2},
$$

$$
\|u(t, \cdot)\|_{B V}, \quad\|u(t, x)\|_{L^{\infty}} \leq c_{0}\left(\left|u_{0}\right|_{B V(R)}+t\left\|u_{0}\right\|_{L^{2}(R)}^{2}\right),
$$

$$
\begin{aligned}
\left\|u_{\varepsilon}(t, \cdot)\right\|_{L^{2 N}(R)} \leq & c_{0}\left(1+\left\|u_{0}\right\|_{L^{2}}^{2}\right)^{2} t \\
& +\|u(0, \cdot)\|_{L^{2 N}(R)}^{2 N} e^{c_{0}\left(1+\left\|u_{0}\right\|_{L^{2}}^{2}\right)^{2} t},
\end{aligned}
$$

where $c_{0}$ is a positive constant independent of t and $\varepsilon$.

The following Oleinik type estimate holds for a.e. $(t, x) \in$ $(0, T] \times R:$

$$
\partial_{x} u(t, x) \leq \frac{1}{t}+K_{T}
$$

where

$$
\begin{aligned}
K_{T}=c_{0}\{ & \left\|u_{0}\right\|_{L^{2}(R)}^{2} \\
& \left.+\left(\left\|u_{0}\right\|_{B V(R)}+T\left\|u_{0}\right\|_{L^{2}(R)}^{2}\right)^{2}\right\}^{1 / 2} .
\end{aligned}
$$

This theorem is an immediate consequence of Theorem 13 and results are presented in Section 2.

Theorem 13 (existence). Assume that $u_{0} \in L^{1}(R) \cap L^{2}(R) \cap$ $B V(R)$ and the solution $u(t, x)$ satisfies (57). Then, there exists at least one weak solution to problem (6).

Proof. Using the estimates obtained in Section 2, we take a standard argument to see that there exists a sequence of strictly positive numbers $\left\{\varepsilon_{k}\right\}_{k=1}^{\infty}$ tending to zero such that as $k \rightarrow \infty$

$$
\begin{gathered}
u_{\varepsilon_{k}} \rightarrow u \quad \text { a.e. in } R_{+} \times R, \\
u_{\varepsilon_{k}} \longrightarrow u \quad \text { in } L_{\text {loc }}^{1}\left(R_{+} \times R\right) .
\end{gathered}
$$

The previous estimates in Section 2 imply immediately that the limit function $u$ satisfies (59)-(60).

Let us now prove that as $k \rightarrow \infty$

$$
P_{\varepsilon_{k}} \longrightarrow P^{u}, \quad \partial_{x} P_{\varepsilon_{k}} \longrightarrow \partial_{x} P^{u} \quad \text { in } L^{1}((0, T) \times R) \forall T>0,
$$

which follows from the following calculations:

$$
\begin{aligned}
& \| P_{\varepsilon_{k}}- P^{u} \|_{L^{1}((0, T) \times R)} \\
& \leq\left(\frac{|m-1|}{4}\right) \\
& \times \int_{0}^{T} \int_{R}\left(\int_{R} e^{-|x-y|}\right. \\
& \leq\left(\frac{|m-1|}{2}\right) \int_{0}^{T}\left(\int_{R}\left|u_{\varepsilon_{k}}(t, y)-u(t, y)\right|\right. \\
& \leq C_{T} \int_{0}^{T} \int_{R}\left|u_{\varepsilon_{k}}(t, y)-(u(t, y))\right| d y d t \longrightarrow 0 \\
&\left.\quad \times \int_{0}^{T} \int_{R}\left(\int_{R} e^{-|x-y|}(t, y)\right)^{2}-(u(t, y))^{2} \mid d y\right) d x d t \\
& \leq\left(\frac{|m-1|}{4}\right) \\
&\left.\left\|\partial_{x} P_{\varepsilon_{k}}-\partial_{x} P^{u}\right\| L_{\varepsilon^{1}\left((0, T)^{1} \times R\right)}(t, y)+u(t, y) \mid d y\right) d t \\
&\left.\leq\left|\left(u_{\varepsilon_{k}}(t, y)\right)^{2}-(u(t, y))^{2}\right| d y\right) d x d t
\end{aligned}
$$




$$
\begin{aligned}
& \leq\left(\frac{|m-1|}{2}\right) \int_{0}^{T}\left(\int_{R}\left|u_{\varepsilon_{k}}(t, y)-u(t, y)\right|\right. \\
& \left.\times\left|u_{\varepsilon_{k}}(t, y)+u(t, y)\right| d y\right) d t \\
& \leq C_{T} \int_{0}^{T} \int_{R}\left|u_{\varepsilon_{k}}(t, y)-(u(t, y))\right| d y d t \rightarrow 0 \\
& \text { as } k \longrightarrow \infty .
\end{aligned}
$$

From (62) to (64), we complete the proof.

\section{Conflict of Interests}

The authors declare that there is no conflict of interests regarding the publication of this paper.

\section{Acknowledgment}

This work is supported by the Fundamental Research Funds for the Central Universities (JBK120504).

\section{References}

[1] A. Constantin and D. Lannes, "The hydrodynamical relevance of the Camassa-Holm and Degasperis-Procesi equations," Archive for Rational Mechanics \& Analysis, vol. 192, no. 1, pp. 165-186, 2009.

[2] A. Degasperis and M. Procesi, "Asymptotic integrability," in Symmetry \& Perturbation Theory, vol. 1, no. 1, pp. 23-37, World Scientific, Singapore, 1999.

[3] J. Escher, Y. Liu, and Z. Y. Yin, "Global weak solutions and blowup structure for the Degasperis-Procesi equation," Journal of Functional Analysis, vol. 241, no. 2, pp. 457-485, 2006.

[4] Y. Fu, Y. Liu, and C. Z. Qu, "On the blow-up structure for the generalized periodic Camassa-Holm and Degasperis-Procesi equations," Journal of Functional Analysis, vol. 262, no. 7, pp. 3125-3158, 2012.

[5] V. O. Vakhnenko and E. J. Parkes, "Periodic and solitary-wave solutions of the Degasperis-Procesi equation," Chaos, Solitons \& Fractals, vol. 20, no. 5, pp. 1059-1073, 2004.

[6] H. Lundmark and J. Szmigielski, "Multi-peakon solutions of the Degasperis-Procesi equation," Inverse Problems, vol. 19, no. 6, pp. 1241-1245, 2003.

[7] D. D. Holm and M. F. Staley, "Wave structure and nonlinear balances in a family of evolutionary PDEs," SIAM Journal on Applied Dynamical Systems, vol. 2, no. 3, pp. 323-380, 2003.

[8] Z. W. Lin and Y. Liu, "Stability of peakons for the DegasperisProcesi equation," Communications on Pure \& Applied Mathematics, vol. 62, no. 1, pp. 125-146, 2009.

[9] Z. Y. Yin, "Global weak solutions for a new periodic integrable equation with peakon solutions," Journal of Functional Analysis, vol. 212, no. 1, pp. 182-194, 2004.

[10] Y. Matsuno, "Multisoliton solutions of the Degasperis-Procesi equation and their peakon limit," Inverse Problems, vol. 21, no. 5, pp. 1553-1570, 2005.

[11] D. Henry, "Infinite propagation speed for the DegasperisProcesi equation," Journal of Mathematical Analysis \& Applications, vol. 311, no. 2, pp. 755-759, 2005.
[12] O. G. Mustafa, "A note on the Degasperis-Procesi equation," Journal of Nonlinear Mathematical Physics, vol. 12, no. 1, pp. 1014, 2005.

[13] Y. Liu and Z. Y. Yin, "Global existence and blow-up phenomena for the Degasperis-Procesi equation," Communications in Mathematical Physics, vol. 267, no. 3, pp. 801-820, 2006.

[14] S. Y. Lai and Y. H. Wu, "The local well-posedness and existence of weak solutions for a generalized Camassa-Holm equation," Journal of Differential Equations, vol. 248, no. 8, pp. 2038-2063, 2010.

[15] Y. Zhou, "Blow-up of solutions to the DGH equation," Journal of Functional Analysis, vol. 250, no. 1, pp. 227-248, 2007.

[16] G. M. Coclite and K. H. Karlsen, "On the well-posedness of the Degasperis-Procesi equation," Journal of Functional Analysis, vol. 233, no. 1, pp. 60-91, 2006.

[17] G. M. Coclite, K. H. Karlsen, and H. Holden, "Wellposedness for a parabolic-elliptic system," Discrete \& Continuous Dynamical Systems, vol. 13, no. 3, pp. 659-682, 2005. 


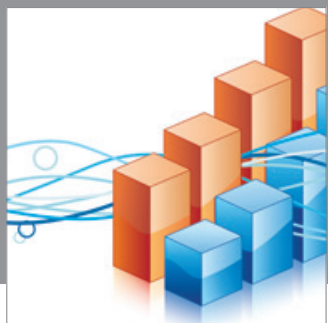

Advances in

Operations Research

mansans

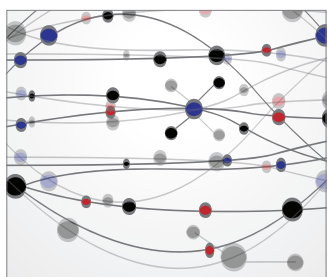

The Scientific World Journal
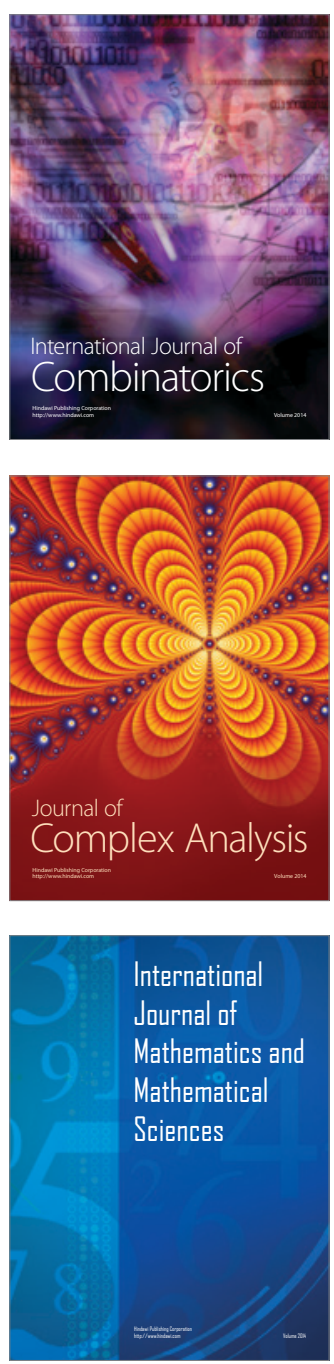
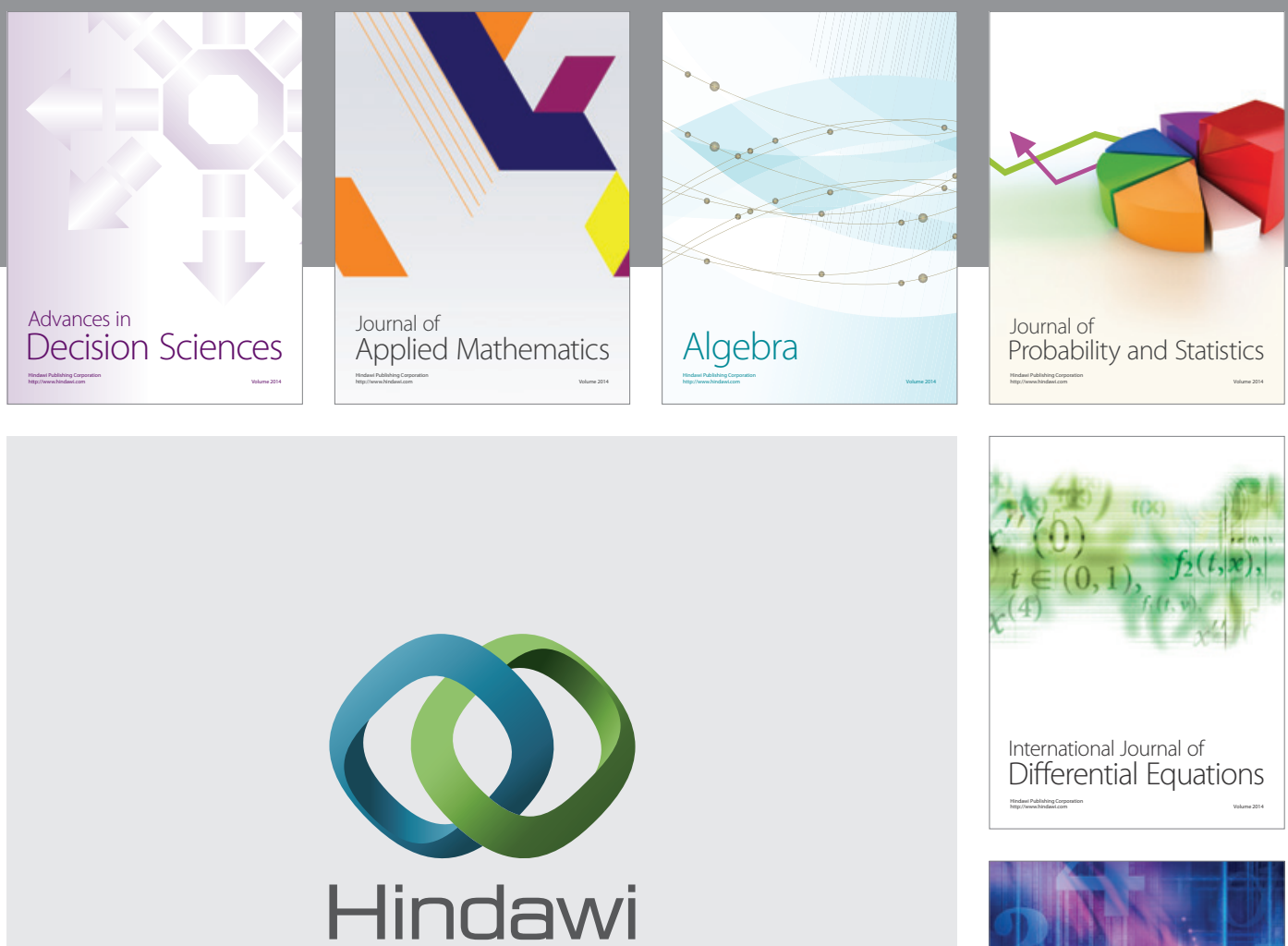

Submit your manuscripts at http://www.hindawi.com
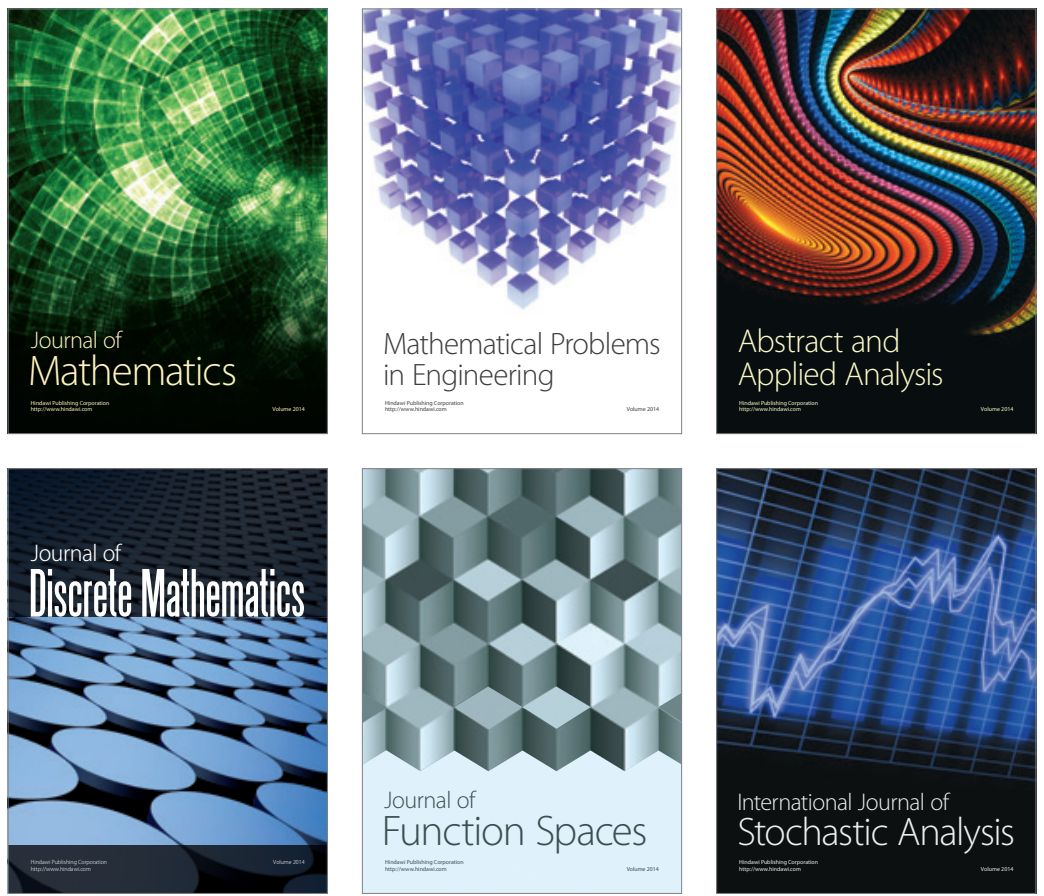

Journal of

Function Spaces

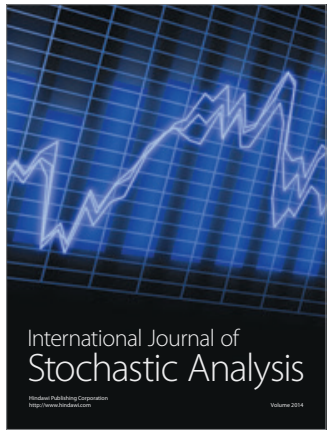

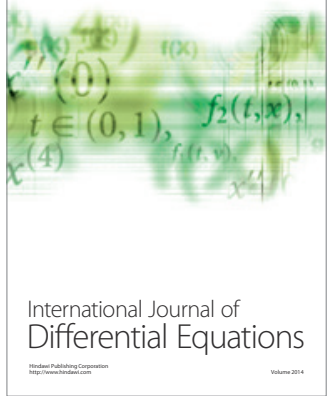
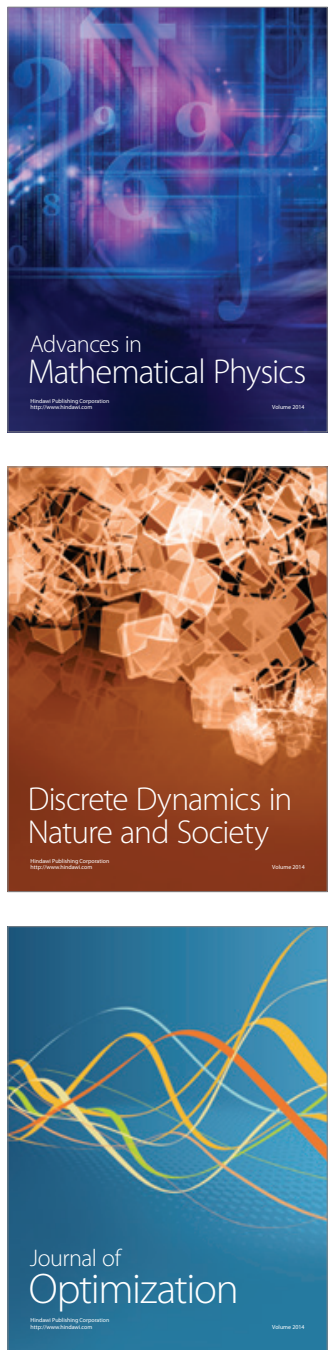